\title{
Die primäre Radiusfrakturprothese bei nicht rekonstruierbaren osteoporotischen distalen Radiusfrakturen
}

Peter Kaiser, Rohit Arora

\section{Einleitung}

Die distale Radiusfraktur gehört zu den häufigsten Brüchen des menschlichen Körpers. Sie tritt grundsätzlich in jedem Alter durch einen Sturz auf das hyperextendierte Handgelenk auf, wobei sich kindliche Formen stark von Formen des Erwachsenen unterscheiden. Bei Kindern kann es zu Wulstbrüchen, Epiphysiolysen, Grünholzfrakturen oder metaphysären Brüchen kommen. Bei Erwachsenen zeigen sich unterschiedlichste Frakturen, angefangen von unverschobenen Brüchen über unterschiedliche Avulsionen und metaphysäre sowie artikuläre Frakturen bis hin zu Luxationsfrakturen. Abhängig von der Kraftrichtung und der Handgelenkposition zum Zeitpunkt des Unfalls kommt es zu einem entsprechenden Verletzungsmuster, wie es eine biomechanische Studie von Pechlaner et al. [1] zeigen konnte. Im Rahmen eines Sturzes kommt es bei gering wirkenden Kräften zu einer elastischen Deformierung bzw. Erweiterung des skapholunären Spaltes, des distalen Radioulnar- und des Mittkarpalgelenkes. Erst bei stärker wirkenden Kräften kommt es zu einer Fraktur im metaphysären Bereich, wobei es in Abhängigkeit von der Kraftrichtung der proximalen Handwurzelreihe auf den Radius zu Colles-artigen (nach dorsal dislozierten) oder Smith-artigen (nach palmar dislozierten) Brüchen bei hyperextendiertem Handgelenk kommt.

Es bestehen unterschiedliche Klassifikationen (z.B. AOKlassifikation, Fernandez-Klassifikation oder PechlanerKlassifikation) der distalen Radiusfraktur, jedoch sind diese oftmals nicht adäquat behandlungsorientiert und inkludieren keine Begleitverletzungen. Hierbei ist als Beispiel die Colles-Fraktur zu nennen. Während diese bei höheren Dislokationsgraden im Erwachsenenalter oftmals operativ versorgt wird, kann eine solche Verletzung bei geriatrischen Patienten trotz Dislokation konservativ mittels Gipsruhigstellung behandelt werden. Hierbei konnte weder in retrospektiven noch in randomisierten prospektiven Studien ein Unterschied im Outcome festgestellt werden [2,3]. Bei geriatrischen Patienten besteht keine Korrelation zwischen den radiologischen Parametern und klinischen Ergebnissen. Ältere Patienten mit Komorbiditäten und geringem funktionellen Bedarf tolerieren Fehlstellungen deutlich mehr. Des Weiteren liegt in dieser Patientengruppe meist eine Osteoporose vor, die einen operativen Eingriff schwieriger gestaltet und Komplikationen häufiger verursacht. Hier ist in erster Linie der
Schrauben- und Repositionshalt zu nennen. Auch winkelstabile Implantate vermögen es aufgrund der metaphysären Trümmerzone nicht, die Reposition aufrecht zu erhalten, sodass es zu einem Repositionsverlust samt konsekutiver intraartikulärer Schraubenlage kommen kann.

Es können im Alter jedoch auch Frakturformen entstehen, die so stark verschoben und destruiert sind, dass eine konservative Therapie einerseits nicht zielführend erscheint und andererseits eine operative Versorgung mittels Plattenosteosynthese, Kirschner-Drähten oder eines externen Fixateurs als unmöglich erachtet werden kann.

Als Beispiel sei hier eine Patientin im Alter von 87 Jahren gezeigt ( $\triangleright$ Abb. 1). Wie die Röntgen- und CT-Bilder anschaulich darstellen, erscheint eine operative Versorgung aufgrund der massiven Dislokation erforderlich. Eine adäquate Rekonstruktion erscheint aufgrund der multifragmentären, intraartikulären Situation unmöglich bzw. ohne voraussehbares, akzeptables Ergebnis. Bei einer entsprechenden Verletzung an anderer Stelle der oberen Extremität, wie bspw. an der Schulter, würde eine Frakturprothese zum Einsatz kommen [4, 5]. Roux verwendet bereits seit 2005 [6] und Herzberg seit 2011 [7] eine Handgelenkhemiprothese, die in solchen Fällen eine Alternative zur Osteosynthese sein kann. Auch bei unserer Patientin wurde mit dem Ziel einer schnellen Rehabilitation samt Erhalt einer guten Funktion, aufgrund bereits genannter Gründe, eine Handgelenkfrakturprothese implantiert ( $\bullet$ Abb. 2). Das Ziel dieses Artikels ist es, die Ergebnisse dieser Handgelenkhemiprothese vorzustellen.

\section{Beschreibung der Prothese [8]}

Bei der Cobra-Prothese (Groupe Lépine, Lyon, Frankreich) handelt es sich um eine Hemiprothese für den distalen Radius ( $\triangleright$ Abb. 3). Sie wurde eigens für ältere Patienten entwickelt, die sich eine nicht rekonstruierbare, multifragmentäre distale Radiusfraktur zugezogen haben. Die Prothese ist als zementierte und zementfreie Version mit einem doppelten Belag aus porösem Titan und Hydroxylapatit für jeweils die rechte und linke Seite verfügbar. Es sind 2 Größen der zementfreien Prothese verfügbar. Im Falle von schlechter Knochenqualität besteht die Möglichkeit, eine zementierte Prothese zu implantieren, um die adäquate Radiuslänge zu gewährleisten. Das Implan- 

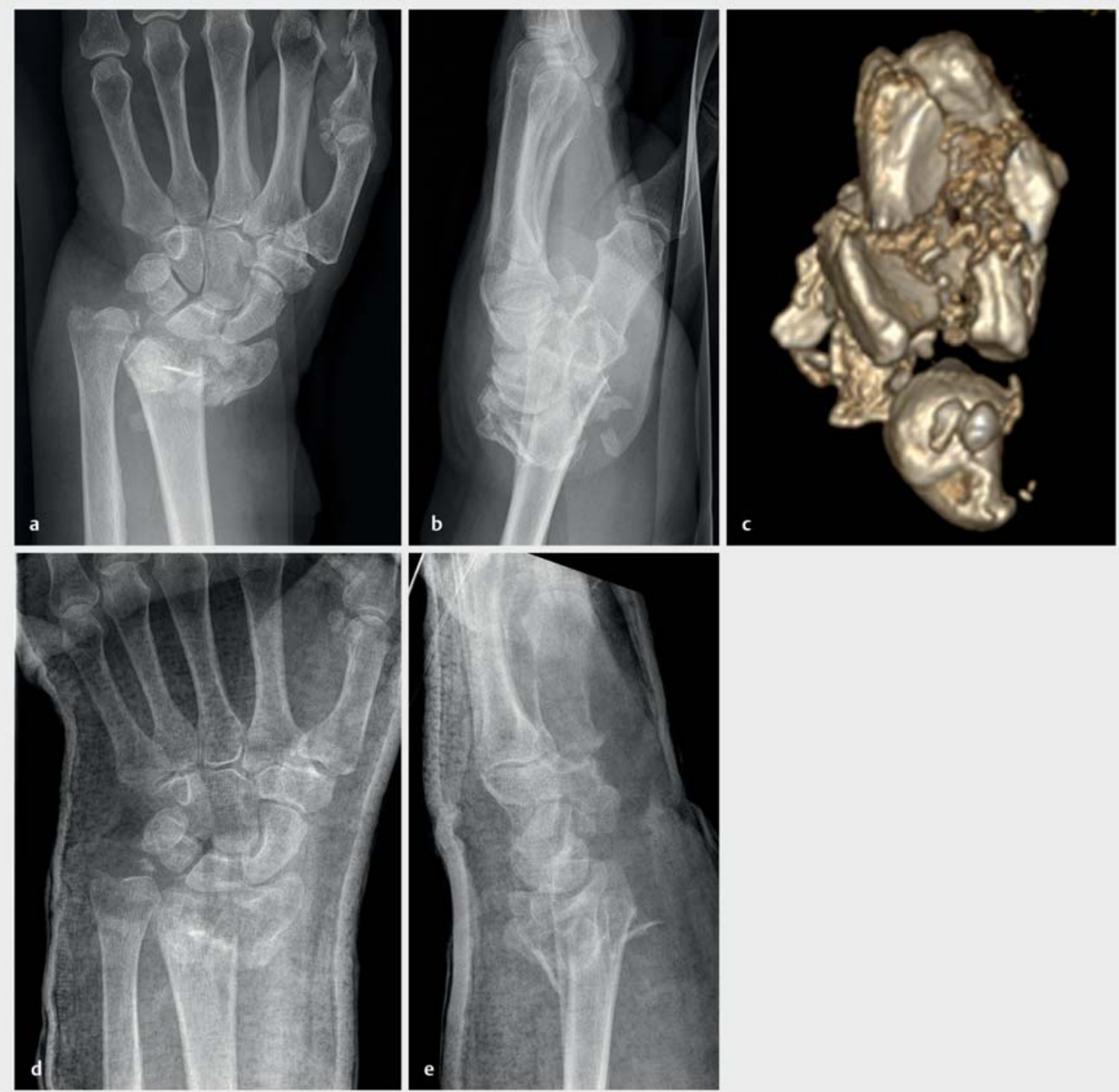

An der radialen und ulnaren Seite der Prothese befinden sich 2 Finnen mit zentralen Löchern, durch die es möglich ist, Knochenfragmente mittels Nähten an die Prothese zu fixieren. Hierdurch wird die Stabilität beim Einbau zusätzlich erhöht.

\section{Beschreibung der Operationsschritte $[7,9]$}

Der Operateur sollte sich distal der Hand befinden, um beim Einbau eine bessere Kontrolle bei der Positionierung

des Implantats in der Transversalebene zu gewährleisten. Der Zugang befindet sich am dorsalseitigen Handgelenk. Eine etwa $8 \mathrm{~cm}$ lange Inzision erfolgt longitudinal in der Mittellinie des Handgelenks in einer Linie mit dem 3. Mittelhandknochen. Das 3. Strecksehnenfach wird longitudinal eröffnet. Als Variante ist auch eine Eröffnung des Strecksehnenretinakulums samt Lappenschnittführung möglich, um einen Retinakulumanteil unterhalb der Extensor-pollicis-longus-Sehne als Gleitschutz beim Verschluss zu platzieren. Im Rahmen des Zugangs muss darauf geachtet werden, die interkarpalen Bänder nicht zu verletzen.

Mittels Osteotom wird die Trümmerfraktur auf Höhe des 3. Strecksehnenfaches buchförmig eröffnet, indem radial 
und ulnar $21 \mathrm{~cm}$ dicke, osteotendinöse Lappen eleviert werden. Der posteriore Interosseousnerv wird im Sinne einer Denervierung hierbei koaguliert und reseziert. Durch eine $1 \mathrm{~cm}$ große Kapselinzision wird die Konvexität der 1. Karpalreihe dargestellt, wobei das dorsale interkarpale und das dorsale skapholunäre Band nicht verletzt werden dürfen. Hiernach wird das Handgelenk auf 70$90^{\circ}$, auf einer Tuchrolle liegend, flektiert und die distalen zertrümmerten, osteokartilaginären Fragmente des distalen Radius exzidiert. Eine periphere Schicht aus Spongiosa und osteoperiostalem Gewebe im palmaren, radialen und dorsalen Anteil wird für die spätere Umgebung des Implantats aufbewahrt.

Die distale Radiusdiaphyse wird mithilfe von 2 HohmannHebeln dargestellt. Der anteriore Anteil der Radiusepiphyse wird mit einem flachen Instrument verfolgt. Dies ist wichtig, da die Ausrichtung des Implantats in der Transversebene parallel zu dieser Landmarke verläuft. Der Markkanal wird mit einer speziellen Raspel parallel zum vorderen Anteil der Radiusepiphyse eingebracht ( Abb. 4). Während dieses Schritts hält der Assistent die Radiusdiaphyse mit einer Knochenklemme in Pronation, sodass der flache Teil der Radiusmetaphyse parallel zum Boden verläuft. Etwa 1,5 cm der Raspel werden aus der Diaphyse herausgelassen, um die Radiuslänge beizubehalten.

Das Probeimplantat wird sanft in den Markraum eingeschlagen. Die beiden Finnen helfen bei der Kontrolle der Positionierung des Implantats in der Transversalebene. Um die Radiuslänge gemäß einer präoperativen Planung mittels Schablonen zu gewährleisten, sollten etwa $1,5 \mathrm{~cm}$ des Implantats aus der Diaphyse herausstehen.

Nun erfolgt die Reposition des Karpus auf das Probeimplantat. Falls die Reposition unmöglich oder zu straff erscheint, sollte das Implantat weiter proximal in die Diaphyse eingeschlagen werden. Falls die Reposition zu locker ist und eine Dislokation zwischen Karpus und Implantat leicht möglich ist, sollte das Implantat weiter aus der Diaphyse herausgelassen werden. Dies kann dadurch erreicht werden, indem etwas Spongiosa in den Markraum eingebracht wird oder indem das endgültige Implantat später in einer geeigneten Länge einzementiert wird.

In jedem Fall sollte die originale Radiuslänge so anatomisch wie möglich wiederhergestellt werden, um eine adäquate Stabilität zwischen dem Implantat und dem Karpus zu gewährleisten. Zwischen dem Implantat und dem Karpus dürfen maximal 2 mm Spiel vorhanden sein.

Falls die knöchernen Fragmente der „sigmoid notch“ zusammengeführt, vernäht und erhalten werden können, bleibt der Ulnakopf intakt und das distale Radioulnargelenk gerettet. Falls die knöchernen Fragmente der „sig-
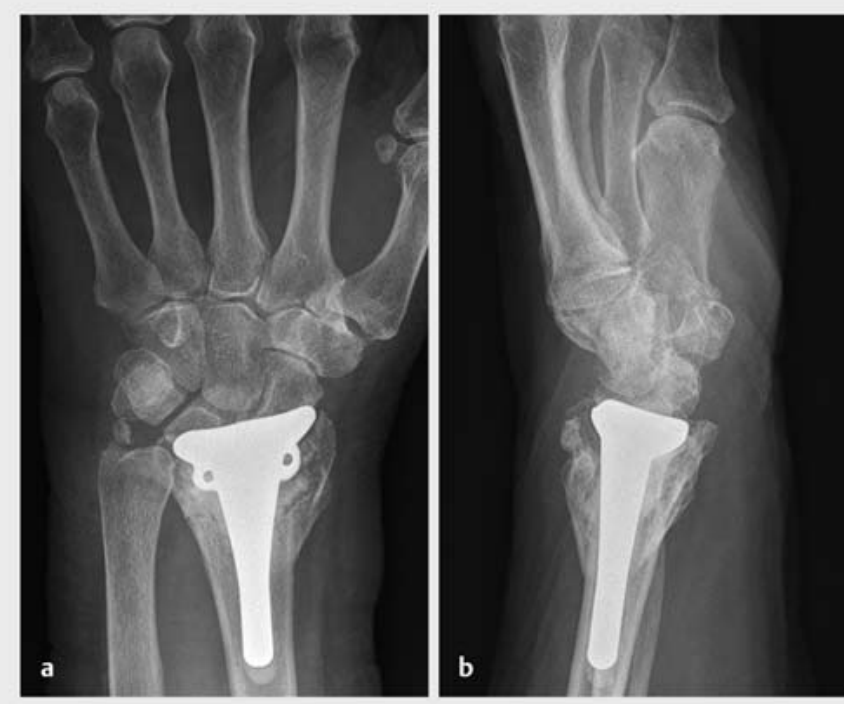

- Abb. 2 Postoperative Darstellung der Patientin aus - Abb. 1. Knapp 1 Jahr postoperativ ist die Patientin sehr zufrieden und präsentiert eine sagittale Bewegung von 30-0-40 , eine freie Rotation sowie einen kompletten Faustschluss.
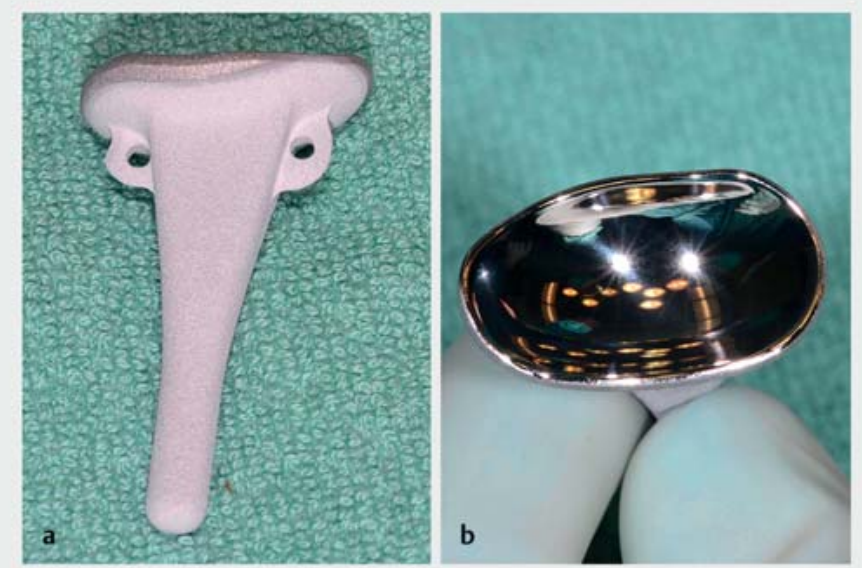

Abb. 3 Cobra-Prothese (Groupe Lépine, Lyon, Frankreich).

moid notch" nicht rekonstruierbar sind oder falls eine Ulnakopf- oder -halsfraktur vorliegt, wird der Ulnakopf nach Watson schräg reseziert $[10,11]$. Der Ulnastumpf wird durch transossäre Nähte der palmaren Kapsel des distalen Radioulnargelenks mit dem dorsalen Anteil des Ulnarstumpfes nach Blatt stabilisiert.

Das endgültige Implantat wird entsprechend der geeigneten Länge sanft in die Diaphyse eingeschlagen. Meistens ergibt sich die Primärstabilität des endgültigen Implantats bei einer geeigneten Länge leicht. Ist dies nicht 

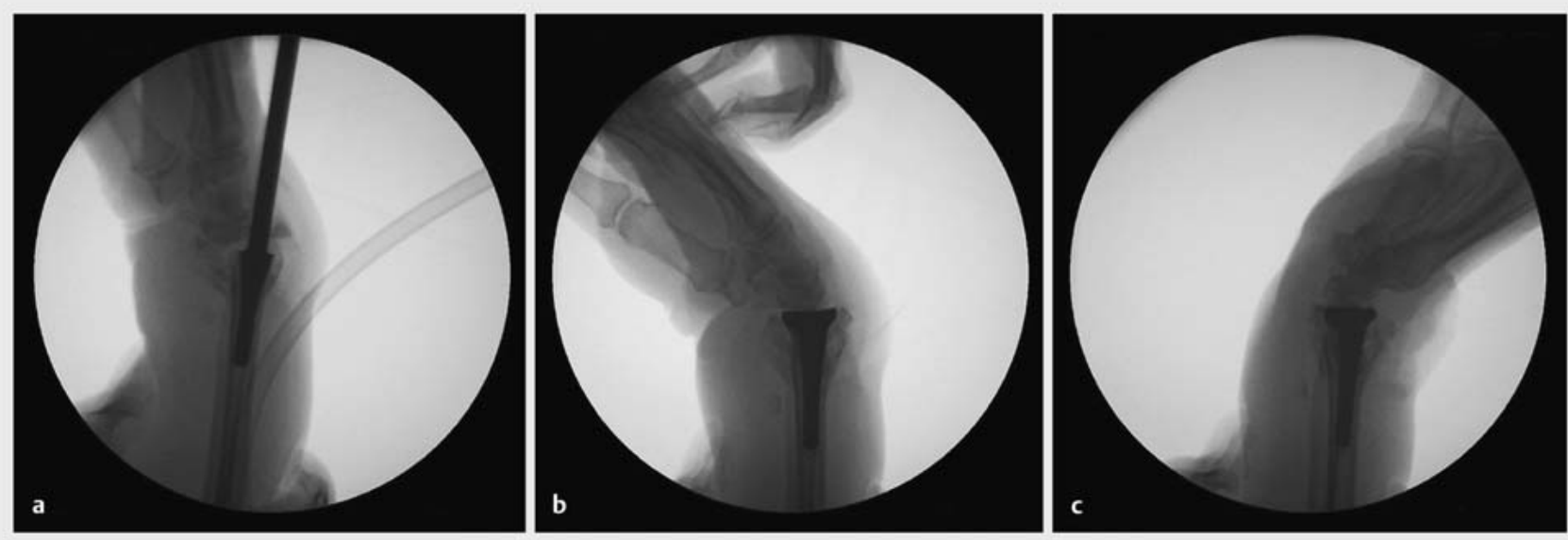

- Abb. 4 Intraoperatives Bild mit der Raspel in der Radiusdiaphyse sowie Bilder der eingebauten Prothese mit maximaler Handgelenkflexion und -extension ohne radiokarpale Subluxationstendenz. Hierdurch kann die Stabilität und die maximal zu erwartende sagittale Beweglichkeit dokumentiert werden.

der Fall, sollte ein zementiertes Implantat verwendet werden. Anschließend wird der Karpus auf das Implantat reponiert. Es erfolgt noch einmal die Überprüfung, ob maximal $2 \mathrm{~mm}$ Spiel zwischen dem Implantat und dem Karpus bestehen.

Die beiden peripheren osteotendinösen Lappen werden wieder zusammengeführt, als würde man ein Buch verschließen, und mit nicht resorbierbaren Nähte zusammengenäht, wobei darauf geachtet werden muss, dass die Strecksehnenfächer nicht mitvernäht werden. Die Extensor-pollicis-longus-Sehne kann aus dem Kompartiment nach subkutan verlagert werden.

Bei Bedarf kann um das Implantat herum freie Spongiosa gelagert werden, um knöcherne Defekte auszufüllen. Knöcherne Fragmente können an die seitlichen Laschen des Implantats angenäht werden. Die kleine Kapselinzision wird mit 2 Nähten mit nicht resorbierbarem Monofilamentnahtmaterial verschlossen.

- Abb. 5 zeigt den intraoperativen Situs vor und nach Prothesenimplantation.

Die Stabilität und die zu erwartende sagittale Beweglichkeit kann intraoperativ mittels eines Bildwandlers dokumentiert werden ( $\triangleright$ Abb. 4).

Postoperativ wird das Handgelenk in einer palmaren Unterarmschiene in Funktionsstellung für 3 Wochen nachbehandelt. Bei zementierten Implantaten wird sofort mit der funktionellen Therapie begonnen. Bei einer unzementierten Implantation wird erst 3 Wochen postoperativ mit Bewegungsübungen samt Unterarmrotation und Handgelenkflexion und -extension begonnen.

\section{Datenlage}

Die Idee der Hemiprothesenimplantation bei älteren Patienten mit einer distalen Radiustrümmerfraktur wurde bereits 2005 durch Roux umgesetzt. Die Implantation der Hemiprothese stellt nämlich eine relativ einfache Methode bei einem komplexen Problem dar. Die klinischen Ergebnisse erscheinen zudem besser und mit einer schnelleren Rehabilitation vergesellschaftet als durch rekonstruktive, osteosynthetische Eingriffe [12] ( Tab. 1).

Das Hemiprothesendesign von Roux hat jedoch einige Nachteile. Einerseits muss sehr viel Knochenmaterial reseziert werden, andererseits ist ein Einbau bei einer gleichzeitigen Ulnakopf- oder -halsfraktur kontraindiziert.

Daher entwickelte Herzberg das hier vorgestellte, knochensparende Design. Er hat seine Erfahrungen in mehreren Publikationen veröffentlicht, wobei die Autoren die Ergebnisse der hier vorgestellten Prothese mit der radialen Komponente der Press-fit-Remotion-Totalprothese (Small Bone Innovations, Morrsiville, PA, USA) kombiniert darstellen. Dadurch können aus den Daten zumindest keine genauen Angaben bez. der hier vorgestellten Prothese herausgefiltert werden ( $\triangleright$ Tab. 2).

Es besteht lediglich eine weitere Studie von Anger et al. aus dem Jahr 2018 [13], welche dieses Prothesendesign an 11 Patienten (weiblich, 65-91 Jahre) mit einem Follow-up von durchschnittlich 18 Monaten retrospektiv evaluiert ( $\vee$ Tab. 2 ). Hierbei wurden 8 Prothesen zementiert und 3 Prothesen zementfrei eingebaut. Insgesamt waren 7 Patienten subjektiv zufrieden oder sehr zufrieden und nur 4 Patienten unzufrieden. Kein Patient zeigte eine 

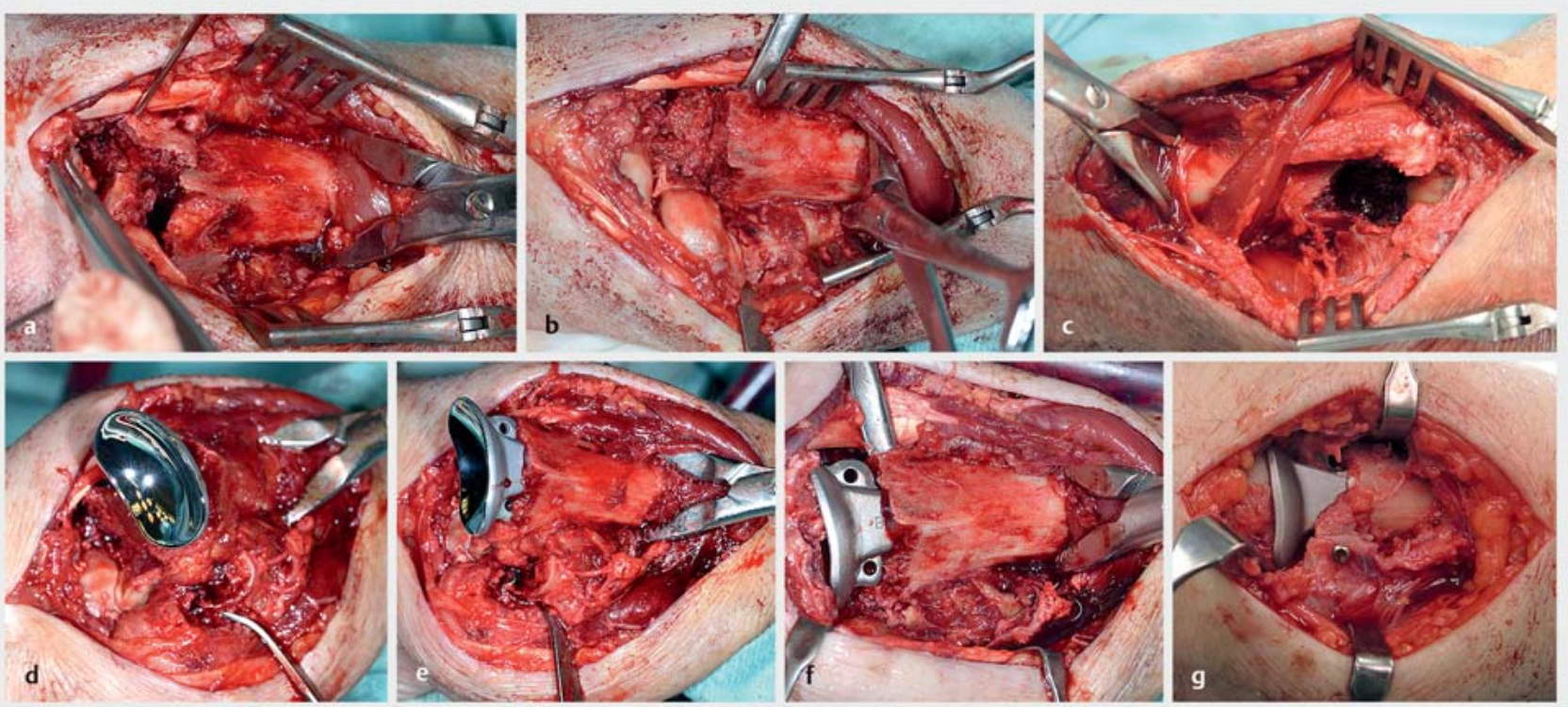

- Abb. 5 Darstellung des intraoperativen Situs vor und nach Exzision der Frakturfragmente sowie Darstellung der Prothese in situ und der radiokarpalen Reposition.

- Tab. 1 Outcome-Ergebnisse des SOPHIA-Prothesendesigns (Biotech International, Salon de Provence, Frankreich).

\begin{tabular}{|l|l|l|l|l|l|l|l|l|l|}
\hline Autoren & $\begin{array}{l}\text { Patien- } \\
\text { tenan- } \\
\text { zahl }\end{array}$ & $\begin{array}{l}\text { Durchschnitt des } \\
\text { Nachuntersu- } \\
\text { chungszeitraumes }\end{array}$ & $\begin{array}{l}\text { Exten- } \\
\text { sion } \\
\text { (Monate) }\end{array}$ & Flexion & $\begin{array}{l}\text { Ulnarab- } \\
\text { duktion }\end{array}$ & $\begin{array}{l}\text { Radial- } \\
\text { abduk- } \\
\text { tion }\end{array}$ & $\begin{array}{l}\text { Pronati- } \\
\text { on }\end{array}$ & $\begin{array}{l}\text { Supina- } \\
\text { tion }\end{array}$ & $\begin{array}{l}\text { Griffkraft (\% } \\
\text { der kontrala- } \\
\text { teralen Seite) }\end{array}$ \\
\hline Roux (2009) [14] & 6 & 27 & 65 & 30 & 20 & 20 & 60 & 50 & 80 \\
\hline Roux (2011) [6] & 10 & 29 & 60 & 36 & 26 & 21 & 67 & 61 & 72 \\
\hline Roux (2016) [12] & 23 & 56 & 62 & 37 & 26 & 31 & 72 & 68 & 79 \\
\hline $\begin{array}{l}\text { Vergnenègre et al. } \\
\text { (2014) [15] }\end{array}$ & 8 & 25 & 44 & 45 & 25 & 20 & 85 & 75 & 92 \\
\hline
\end{tabular}

- Tab. 2 Outcome-Ergebnisse des Cobra-Prothesendesigns (Groupe Lépine, Lyon, Frankreich).

\begin{tabular}{|c|c|c|c|c|c|c|c|c|c|c|}
\hline Autoren & $\begin{array}{l}\text { Patien- } \\
\text { tenan- } \\
\text { zahl }\end{array}$ & $\begin{array}{l}\text { Durchschnitt des } \\
\text { Nachuntersu- } \\
\text { chungszeitrau- } \\
\text { mes (Monate) }\end{array}$ & $\begin{array}{l}\text { Schmerz } \\
\text { (VAS) }\end{array}$ & $\begin{array}{l}\text { Quick- } \\
\text { DASH } \\
\text { Score }\end{array}$ & $\begin{array}{l}\text { PRWE } \\
\text { Score }\end{array}$ & $\begin{array}{l}\text { Lyon } \\
\text { Wrist } \\
\text { Score }\end{array}$ & $\begin{array}{l}\text { Unter- } \\
\text { armro- } \\
\text { tation }\end{array}$ & $\begin{array}{l}\text { sagit- } \\
\text { tale Be- } \\
\text { weglich- } \\
\text { keit }\end{array}$ & $\begin{array}{l}\text { Exten- } \\
\text { sion }\end{array}$ & $\begin{array}{l}\text { Griffkraft } \\
\text { (\% der kon- } \\
\text { tralateralen } \\
\text { Seite) }\end{array}$ \\
\hline $\begin{array}{l}\text { Herzberg et al. } \\
(2015)^{*}[7]\end{array}$ & 11 & 27 & 1 & 32 & 25 & 73,1 & 151 & 60 & 34 & 67 \\
\hline $\begin{array}{l}\text { Herzberg et al. } \\
(2017)^{*}[16]\end{array}$ & 12 & 32 & 1 & 25 & 22 & 75 & 149 & 62 & 35 & 69 \\
\hline $\begin{array}{l}\text { Herzberg et al. } \\
(2018)^{*}[10]\end{array}$ & 27 & 32 & 1 & 26 & 25 & 74 & 150 & 60 & 36 & 68 \\
\hline $\begin{array}{l}\text { Anger et al. (2018) } \\
\text { [13] }\end{array}$ & 11 & 18 & 3,8 & 59 & 72 & 50 & 164 & 63 & 27 & 44 \\
\hline
\end{tabular}

${ }^{*}$ Ergebnisse kombiniert mit der radialen Komponente der Press-fit-Remotion-Totalprothese (Small Bone Innovations, Morrsiville, PA, USA) 
Implantatlockerung oder -dislokation. Radiologisch konnte auch kein radialer oder ulnarer Tilt des Karpus beobachtet werden. Es kam zu keiner Infektion, jedoch zeigten 2 Patientinnen ein komplexes regionales Schmerzsyndrom (CRPS). Eine weitere Patientin wurde aufgrund von Schmerzen bei Pronations-Supinations-Bewegungen im distalen Radioulnargelenk nach 6 Monaten mittels einer Ulnakopfresektion erneut operiert, wobei die Beschwerden nicht sistierten.

Die Autoren kommen zum Schluss, dass aufgrund der mittelgradigen Resultate die Hemiprothesenimplantation zwar keinen Goldstandard darstellt, aber dennoch eine Berechtigung in der Therapie von distalen Radiustrümmerfrakturen bei älteren Patienten hat. Es fehlen jedoch noch Langzeitergebnisse, welche die Nützlichkeit der Hemiprothesenimplantation als Therapie bei distalen Radiustrümmerfrakturen bei älteren Patienten bekräftigen.

\section{Konklusion}

Die Hemiprothese stellt zurzeit keinen Goldstandard bei der Therapie von Trümmerfrakturen des distalen Radius bei älteren Patienten dar, jedoch erweitert sie das operative Repertoire und kann in Einzelfällen eine gute Anwendung in der Therapie finden.

\section{Interessenkonflikt}

Die Autorinnen/Autoren geben an, dass kein Interessenkonflikt besteht.

\section{Autorinnen/Autoren}

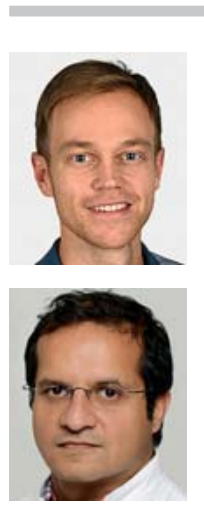

\section{Peter Kaiser}

Dr. med. univ., PhD, Facharzt für Orthopädie und Traumatologie, Department für Unfallchirurgie, Medizinische Universität Innsbruck, Österreich

\section{Rohit Arora}

Assoz. Prof. Priv.-Doz. Dr., Interimistischer Primar, Department für Unfallchirurgie, Medizinische Universität Innsbruck, Österreich

\section{Korrespondenzadresse}

Assoz. Prof. Priv.-Doz. Dr. Rohit Arora

Medizinische Universität Innsbruck

Anichstraße 35

6020 Innsbruck

Österreich

Tel.: + 43/(0)512/504-22825

Fax: + 43/(0)5 12/504-28245

rohit.arora@i-med.ac.at

\section{Literatur}

[1] Pechlaner S, Kathrein A, Gabl M et al. [Distal radius fractures and concomitant lesions. Experimental studies concerning the pathomechanism]. Handchir Mikrochir Plast Chir 2002; 34: $150-157$

[2] Arora R, Lutz M, Deml C et al. A prospective randomized trial comparing nonoperative treatment with volar locking plate fixation for displaced and unstable distal radial fractures in patients sixty-five years of age and older. J Bone Joint Surg Am 2011; 93: 2146-2153

[3] Arora R, Gabl M, Gschwentner M et al. A comparative study of clinical and radiologic outcomes of unstable Colles type distal radius fractures in patients older than 70 years: nonoperative treatment versus volar locking plating. J Orthop Trauma 2009; 23: $237-242$

[4] Erdle B, Izadpanah $\mathrm{K}$, Eberbach $\mathrm{H}$ et al. [Primary fracture prostheses and reverse shoulder arthroplasty in complex humeral head fractures: an alternative to joint-preserving osteosynthesis?]. Orthopade 2018; 47: 410-419

[5] McLean AS, Price N, Graves S et al. Nationwide trends in management of proximal humeral fractures: an analysis of 77,966 cases from 2008 to 2017. J Shoulder Elbow Surg 2019; 28: 2072-2078

[6] Roux JL. Treatment of intra-articular fractures of the distal radius by wrist prosthesis. Revue de Chirurgie Orthopédique et Traumatologique 2011; 97 (Suppl.): S46-S53

[7] Herzberg G, Burnier M, Marc A et al. Primary wrist hemiarthroplasty for irreparable distal radius fracture in the independent elderly. J Wrist Surg 2015; 4: 156-163

[8] Groupe Lépine. Distal Radius Prosthesis COBRA ${ }^{\text {TM }}$. Im Internet: https://www.groupe-lepine.com/en/hand/208-distal-radiusprosthesis-cobra.html; Stand: 09.12.2019

[9] Groupe Lépine. Distal Radius Prothesis COBRA - Surgical Technique. Genay: Groupe Lépine; 2019

[10] Herzberg G, Walch A, Burnier M. Wrist hemiarthroplasty for irreparable DRF in the elderly. Eur J Orthop Surg Traumatol 2018; 28: 1499-1503

[11] Watson HK, Ryu JY, Burgess RC. Matched distal ulnar resection. J Hand Surg Am 1986; 11: 812-817

[12] Roux JL. Wrist hemiarthroplasty for distal radius fractures: 10 years of experience. Hand 2016; 11 (Suppl.): 45S-46S

[13] Anger F, Legré R, Nguyen MK. Results of wrist hemiarthroplasty for comminuted distal radius fractures in independent elderly people: a retrospective study on eleven patients. Hand Surg Rehabil 2019; 38: 150-156

[14] Roux JL. [Replacement and resurfacing prosthesis of the distal radius: a new therapeutic concept]. Chir Main 2009; 28: 1017

[15] Vergnenègre G, Mabit C, Charissoux JL et al. Treatment of comminuted distal radius fractures by resurfacing prosthesis in elderly patients. Chir Main 2014; 33: 112-117

[16] Herzberg G, Merlini L, Burnier M. Hemi-arthroplasty for distal radius fracture in the independent elderly. Orthop Traumatol Surg Res 2017; 103: 915-918

Bibliografie

DOI https://doi.org/10.1055/a-1022-1622 online publiziert 20.01.2020 | OP-JOURNAL 2020; 36: 133138 ๑ Georg Thieme Verlag KG Stuttgart · New York ISSN 0178-1715 